\title{
Avanços na Psicologia Positiva: Bem-Estar, Engajamento e Redesenho no Trabalho
}

\author{
Ana Claudia Souza Vazquez ${ }^{1}$ \\ Universidade Federal de Ciências da Saúde de Porto Alegre, Porto Alegre-RS, Brasil \\ Maria Cristina Ferreira \\ Universidade Salgado de Oliveira , Goiânia-GO, Brasil \\ Helenides Mendonça \\ Pontifícia Universidade Católica de Goiás, Goiânia-GO, Brasil
}

\section{RESUMO}

Baseados na perspectiva da complexidade do comportamento humano, avanços científicos têm sido obtidos por pesquisadores da Psicologia Positiva ao identificar fatores de saúde e de proteção das pessoas em concomitância com os de risco de dano ou agravo á saúde. Esse artigo teórico visa demonstrar o impacto desses achados para a Psicologia Organizacional e do Trabalho, com base em três conceitos centrais. São apresentados no artigo os modelos teóricos que fundamentam as evidências e os principais achados nos estudos sobre bem-estar, engajamento e redesenho do trabalho. A principal contribuição é que as pesquisas estão sendo impulsionadas com modelos integrativos sobre esses fenômenos e suas associações com variáveis de felicidade, proatividade e realização profissional, dentre outras. Somada ao conhecimento dos estudos tradicionais, a Psicologia Positiva possibilita a visão sistêmica sobre trabalho e organizações, tratando a saúde e o bem-estar como premissas do contexto laboral e o desempenho saudável como desfecho positivo das atividades profissionais. Palavras-chave: psicologia positiva; saúde do trabalhador; bem-estar; engajamento no trabalho; comportamento de redesenho no trabalho.

\section{ABSTRACT - Advances in Positive Psychology: Well-Being, Engagement and Job Crafting}

Based on the perspective of the complexity of human behavior, scientific advances are being obtained in Positive Psychology by identifying protective factors concurrently with those that cause risk or harm to health. This theoretical article aims to demonstrate the influence of these findings on organizational and work psychology, based on evidence produced in three central constructs. We present the theoretical models that support the evidence and main findings of studies on well-being, engagement and job crafting. The main contribution is that the studies are being driven with integrative models on these phenomena and their associations with happiness, proactivity and professional achievement variables, among others. Added to the knowledge of the traditional studies, Positive Psychology enables a systemic view of work and organizations, treating health and well-being as premises of the work context and healthy performance as a positive outcome of professional activities.

Keywords: positive psychology; worker's health; well-being; work engagement; job crafting.

\section{RESUMEN - Avances en la Psicología Positiva: bienestar, rendimiento y Job Crafting}

Con base en la perspectiva de la complejidad del comportamiento humano, avances científicos están siendo obtenidos por los investigadores de la psicología positiva al identificar factores de salud y de protección de las personas en concomitancia con los riesgos de daño o agravio de la salud. Este artículo teórico tiene como objetivo demostrar su impacto en la psicología organizacional y laboral, basada en tres constructos centrales. Se presentan modelos teóricos que respaldan las evidencias y los principales hallazgos en los estudios sobre bienestar, rendimiento y job crafting. La principal contribución es que las integraciones están siendo impulsadas con modelos integradores sobre estos fenómenos y sus asociaciones con las variables de felicidad, proactividad, logro profesional, entre otros. Además del conocimiento de los estudios tradicionales, la psicología positiva permite una visión sistémica del trabajo y las organizaciones, tratando la salud y el bienestar como premisas del contexto laboral y el rendimiento saludable como un resultado positivo de las actividades profesionales.

Palabras clave: psicología positiva; salud del trabajador; bienestar; rendimiento; job crafting.

O objetivo desse artigo teórico é demonstrar os avanços em Psicologia Positiva organizacional e do trabalho obtido por meio do desenvolvido de três conceitos principais. Teorias sobre bem-estar, engajamento no trabalho e comportamentos do redesenho do trabalho ganharam campo por seus robustos achados (Mendonça \& Silva, 2019; Schaufeli, 2018). Com o desenvolvimento do campo da Psicologia Positiva Organizacional e do 
Trabalho (PPOT), os pesquisadores passam a analisar também os fatores psicossociais com potencial protetivo e as variáveis latentes para promoção da saúde do trabalhador. Na gestão de pessoas, esse movimento positivo se deu pelo direcionamento das intervenções para aspectos que impulsionam a motivação, o comportamento producente e o desempenho adequado não apenas à produção, mas também à saúde do trabalhador (Vazquez \& Schaufeli, 2019; Nery, Neiva, \& Mendonça, 2016).

O conceito de trabalho e de organizações se transformou pela identificação da complexidade desses fenômenos ao se levar em conta também as características positivas das pessoas, de seu comportamento, de suas relações e do contexto organizacional de sua prática profissional (Dalanhol, Machado, Pizarro, Hutz, \& Vazquez, 2017; Mendonça, Ferreira, Caetano, \& Torres, 2014; Oliveira \& Ferreira, 2016). O movimento da Psicologia Positiva fez com que diferentes conceitos ganhassem espaço no campo científico, tais como resiliência, engajamento, florescimento, gratidão, compaixão, otimismo, perdão. Sem desconsiderar os fatores ou desfechos negativos do trabalho, importantes ganhos dos estudos sobre insatisfação laboral, Burnout, violência no trabalho, dentre outros, são considerados em conjunto com a análise das variáveis positivas (Mendonça \& Ferreira, 2018; Vazquez, Pianezolla, \& Hutz, 2018).

Como uma visão ampliada em saúde, destaca-se noção de "balanceamento ou funcionamento ótimo", expressão sobre o desfecho mais positivo que é consequente das forças e tensões na força de trabalho, em determinado contexto organizacional (Efrom, Vazquez, \& Hutz, 2019; Schaufeli, Bakker, \& Van Rhenen, 2009). Nessa linha, emergiram conceitos singulares como o de florescimento no trabalho, que é uma experiência de equilíbrio ótimo do funcionamento psicológico, em que a pessoa se sente capaz de desenvolver suas potencialidades e de controlar sua vida privada e profissional com vitalidade e emoções positivas (Mendonça \& Ferreira, 2018). O campo da saúde do trabalhador, incluindo a saúde ocupacional, foram pioneiros nessa área, como será demonstrado a seguir.

\section{Saúde do Trabalhador e Psicologia Positiva}

Estudos tradicionais em saúde do trabalhador têm como base dois modelos teóricos principais, desdobrados em múltiplas abordagens e perspectivas (Vazquez, 2018). A teoria Effort-Reward Imbalance (ERI) argumenta que trabalhadores que se envolvem no máximo de tarefas, pela intensa identificação com a organização, desprezam sinais constantes de desgaste físico, psicológico ou emocional que podem levá-los à exaustão ou ao afastamento laboral. Explica essa troca social entre trabalhadores e organização como overcommitment, termo para o qual não há uma tradução precisa e que deve ser entendido como uma overdose de comprometimento. Pessoas com overcommitment se queixam de insatisfação e pressão por demandas laborais, mas permanecem na organização em que desenvolveram suas carreiras, mesmo quando têm uma proposta concreta e promissora para sair (Violanti et al., 2018).

E a teoria Job-Demand-Control (JDC) defende que elevadas demandas psicológicas dificultam ao trabalhador viver de acordo com suas convicções (Job Demand), enquanto a menor amplitude de decisão o constrange em não demonstrar seu conhecimento ou em exercer seu papel nas decisões diárias (Job Control). A carência de suporte social no trabalho aumenta sua vulnerabilidade para situações de violência, assédio e Burnout. Estudos na teoria JDC apontam para modelos de gestão com elevada padronização, cujo modo de organização do trabalho promove o aumento de sintomas musculoesqueléticos por elevação do ritmo laboral, falta de intervalos regulares de recuperação, ciclo reduzido de tempo, demandas emocionais exaustivas, redução de recursos e repetição excessiva de tarefas. Tais sintomas se exacerbam quando o controle do trabalhador é baixo e não há suporte social dos colegas e supervisores no trabalho (Koukoulaki, 2014; Hutz, Zanon, \& Brum Neto, 2013).

Ao longo do tempo e evidências, diversos estudos optaram pela combinação entre as duas teorias vigentes, analisando o desequilíbrio nas trocas sociais com o controle do trabalho. Destaca-se aqui o movimento da salutogênese no campo da saúde do trabalhador, que investiga aspectos da promoção de saúde pela consideração de múltiplos fatores (condições ambientais, aspectos ergonômicos), pela associação entre eles e por suas implicações em qualidade de vida. Aqui, ressaltam-se os conceitos de senso de coerência, workability e elasticity. Em situações adversas de ameaça ou tensão, as pessoas regulam suas percepções criando um senso de coerência pela coesão do sentido atribuído ao evento de vida, que as tornam mais confiantes de que possuem recursos suficientes. Workability é o senso de competência em sua capacidade laboral, que pode predizer tanto a incapacidade futura quanto a duração do absenteísmo por saúde. E elasticity é quanto à organização se adapta às mudanças, sem perder produção, pelo controle das demandas laborais e pelo maior nível de satisfação com as recompensas (Mazloumi, Rostamabadi, Nasl Saraji, \& Rahimi Foroushani, 2012; Magnavita, 2012).

Há muitas evidências na identificação de adoecimentos laborais e sua associação com doenças musculoesqueléticas, cardiovasculares, depressão, estresse crônico, dentre outras (Violanti et al., 2018; Koukoulaki, 2014; Hutz et al., 2013). Tradicionalmente, elas se centram no dano, sofrimento ou injúria ao trabalhador, mesmo na salutogênese, ou seja, o foco está nos desfechos negativos e desfavoráveis à saúde do trabalhador. Recentemente, a Psicologia Positiva influenciou uma terceira via de análise em o que o trabalho é visto como um processo de ativação de energia do trabalhador, associada aos níveis de prazer ou desprazer percebidos ao longo da produção laboral (Salanova, del Libano, Llorens, \& Schaufeli, 
2014). A premissa central dessa teoria é que a associação da ativação de energia laboral com o prazer conduz a comportamentos producentes e ao desenvolvimento positivo. Esse processo tem como premissa o bem-estar e a saúde dos trabalhadores, sendo a base dessa análise a teoria Job-Demand-Resources (JDR). O JDR identifica as demandas e os recursos acessíveis no contexto de trabalho como fatores que se combinam nas práticas profissionais e geram desgaste de energia laboral. Para que se tenha prazer e desfechos positivos, essa energia deve ser recarregada adequadamente, tendo como indicador de saúde o e engajamento no trabalho (Efrom \& Magnan, 2018; Schaufeli, 2018).

Destacam-se, nesse artigo, três conceitos teóricos que detalham o papel da Psicologia Positiva e seu desenvolvimento nas pesquisas e na prática profissional. Bem-estar, engajamento e redesenho do trabalho são construtos centrais pelas robustas evidências obtidas e seu impacto na saúde do trabalhador e no desempenho profissional.

\section{Bem-estar e Felicidade no Trabalho}

Os estudos sobre bem-estar emergiram com duas abordagens distintas, sendo uma objetiva e outra subjetiva. A perspectiva objetiva estuda as características de higiene e segurança enquanto a subjetiva engloba componentes afetivos, cognitivos e psicológicos. No campo afetivo, há dimensões relacionadas às emoções, sendo que o componente cognitivo privilegia a avaliação que o indivíduo faz da própria vida, e o bem-estar psicológico analisa o funcionamento psíquico. No conjunto, os aspectos subjetivos do bem-estar englobam o afeto, a satisfação de vida e a felicidade, sobre como as pessoas experienciam suas vidas positivamente, na perspectiva hedonista (Diener, Oishi, \& Lucas, 2003). E também os conceitos de autoaceitação, autonomia, propósito de vida, crescimento pessoal e relações positivas com os outros, na perspectiva eudaimonista (Ryff, 1989).

Numa perspectiva multifacetada, evidências defendem que o bem-estar pode ser mais bem conceituado como um fenômeno multidimensional, constituído por aspectos hedonistas como eudaimonistas. Ryff (1989) discrimina entre bem-estar subjetivo e bem-estar psicológico. Nesse artigo, defini-se bem-estar psicológico em termos cognitivos, compreendidos pelas competências individuais para lidar com situações concretas da vida. Esse modelo é fundamentado na perspectiva eudaimonista, que significa a busca de excelência pessoal como motivação central da existência, estruturada por seis dimensões: autonomia, para ter um self determinado e independente, capaz de realizar autoavaliações; propósito de vida, nos objetivos que o indivíduo tem na vida e seu senso de direção; domínio do ambiente, para ser capaz de administrar atividades complexas; crescimento pessoal, para ser capaz de manter o próprio processo de desenvolvimento; autoaceitação, como capacidade de aceitação de si e dos outros; relações positivas com outros, de satisfação, de confiança e de afetividade.

Dagenais-Demarais, Mendonça, Ferreira e Savoie (2017) analisam que os estudos em bem-estar no trabalho têm sido influenciados principalmente por três principais abordagens: teorias das emoções, Psicologia Humanística e Psicologia Positiva. Na teorias das emoções, os debates se intensificaram nos anos 1980, ao conceber o bem-estar a partir da razão entre afetos positivos e negativos. Já a Psicologia Humanística foi fundamental na compreensão do bem-estar no trabalho pelas premissas sobre confiança nos seres humanos e em sua bondade fundamental, com foco na experiência subjetiva, autorrealização, congruência e autodeterminação, o que coloca o indivíduo em posição de fazer suas próprias escolhas. Essa influência decorre dos célebres trabalhos de Elton Mayo e Maslow, com as teorias das relações humanas e a teoria das necessidades e motivações, respectivamente.

Por fim, o advento da Psicologia Positiva foi um marco na evolução do conceito de bem-estar, em que os aspectos positivos da experiência humana e os estados psicológicos positivos ganharam destaque. A Psicologia Positiva e o movimento do comportamento organizacional positivo trazem esse objeto de estudo para o campo dos psicólogos organizacionais e do trabalho, em um contexto social em que as próprias relações de trabalho mudam drasticamente (Vazquez, 2018). Nesse hall de conceitos influenciadores, estão: bem-estar, felicidade e florescimento, assim como o engajamento e os comportamentos de redesenho do trabalho. Estudiosos têm enfatizado a necessidade de modelos de bem-estar que os diferenciem da análise de traços ou estados psicológicos (Simmons, 2014).

Apesar dos avanços nos estudos sobre bem-estar no trabalho, os modelos conceituais de referência ainda não agregam a complexidade do construto e a amplitude de sua relevância. Isso decorre, do fato, de alguns autores apresentam bem-estar como um traço estável em vários domínios da vida, incluindo o trabalho ( $\mathrm{Li}$, Yin, Jiang, Wang, \& Cai, 2014), não contemplando as especificidades inerentes que devem ser levadas em conta por pesquisadores e profissionais (Kashdan, Biswas-Diener, \& King 2008). Ademais, alguns indicadores de bem-estar no trabalho não estão suficientemente representados na literatura organizacional.

Dagenais-Desmarais e Savoie (2012) desenvolveram, na abordagem etno-semântica, o modelo de bem-estar psicológico no trabalho, com cinco dimensões: relações interpessoais no trabalho, prosperidade no trabalho, sensação de competência no trabalho, reconhecimento percebido no trabalho e desejo de envolvimento no trabalho. Estas dimensões podem ser reagrupadas sob um único fator de bem-estar no trabalho. A teoria se diferencia por refletir a experiência subjetiva dos trabalhadores sobre o seu próprio bem-estar, uma experiência em que aspectos eudaimônicos se sobressaem a fatores 
hedônicos. Conceitualmente, essa perspectiva integrada parece ser a mais promissora por analisar bem-estar como traço estável e também como estado emocional transitório, que sofre influência das dinâmicas sociais, dos contextos de trabalho e das relações interpessoais.

Pesquisas científicas têm demonstrado o papel das emoções no trabalho para promoção da saúde e incremento da produtividade, sendo o bem-estar um preditor de importante dos resultados organizacionais positivos. Duas teorias são a base dessas discussões. A teoria dos eventos afetivos sugere que o contexto de trabalho predispõe a ocorrência de diferentes eventos - positivos ou negativos - no dia a dia e que eles estimulam reações emocionais respectivas (Weiss \& Cropanzano, 1996). A Broaden and Built Theory por sua vez, estabelece que as emoções positivas têm valor adaptativo com potencial de ampliar os repertórios mentais que levam à ação, por meio da expansão das matrizes de pensamentos (Fredrickson \& Losada; 2005). Em conjunto, essas teorias aprofundam a compreensão de que os eventos diários no trabalho influenciam o bem-estar dos trabalhadores de maneira distinta, a depender das avaliações cognitivas individuais. Reações emergem não apenas pelo evento em si, mas também das avaliações e da forma como o trabalhador enfrenta os problemas cotidianos. Nessa linha, as flutuações no bem-estar devem ser consideradas, colocando esse construto em uma perspectiva distinta das teorias que o consideram traço mais estável de personalidade.

A avaliação do bem-estar no trabalho, com instrumentos de qualidade psicométrica adequados, está descrito em Mendonça e Silva (2019). As avaliações e diagnósticos do bem-estar são extremamente úteis no contexto organizacional, que clama por ajustes e mudanças. Assim é que iniciativas gerenciais focadas no estímulo à produtividade devem considerar a saúde do trabalhador. Torna-se fundamental que os gestores estimulem o bem-estar no trabalho quando têm como foco introduzir metas que estimulam a melhoria no desempenho.

\section{Engajamento no Trabalho}

Estudos na área organizacional e do trabalho têm contribuído para compreender as forças positivas que podem conduzir ao alto desempenho e à realização profissional, com dados relevantes para fomento de estratégias de ação em gestão de pessoas, na prevenção de riscos psicossociais laborais e na promoção de saúde do trabalhador (Timms \& Brough, 2013; Reijseger, Schaufeli, Peeters, \& Taris, 2012). Na Psicologia Positiva, o engajamento no trabalho é definido como um estado mental positivo que desencadeia a sensação de bem-estar, preenchimento e identificação em relação ao trabalho. É um estado mental disposicional, portanto refere-se a motivos intrínsecos que se mantêm ao longo do tempo nas pessoas e que as impulsionam ao comportamento de investir intensa energia e dedicação no trabalho que realizam, com elevada sensação de prazer e realização profissional (Bakker \& Schaufeli, 2008; Vazquez \& Schaufeli, 2019). Diferencia-se da adição ao trabalho (workaholism) por sua conexão com o prazer intenso e a sensação de preenchimento profissional. $\mathrm{Na}$ adição ao trabalho, o elevado investimento de energia e dedicação é um comportamento compulsivo, no qual o prazer não é relevante para esse trabalhador (Taris \& Schaufeli, 2016; Salanova et al., 2014).

Há diferentes pesquisadores e consultores que utilizam o conceito de engajamento, em distintas abordagens, não obstante, a teoria de referência em pesquisas internacionais é Job-Demand Resources Model (JDR) (Schaufeli, 2018). Nessa linha, engajamento no trabalho é o mediador das variáveis de contexto laboral (recursos e demandas, recursos individuais) com o desempenho; tendo sido evidenciado como indicador de saúde do trabalhador, pois o alto investimento de energia atua como fator de proteção e se alia ao intenso prazer em sua atividade profissional (Freitas \& Reis, 2019; Vazquez \& Schaufeli, 2019).

Taris \& Schaufeli (2016) demostram que o modelo JDR é capaz de compreender o que energiza os trabalhadores, quais aspectos incentivam sua persistência no alcance de resultados e quais recursos (pessoais e de trabalho) fazem com que se concentrem mais intensamente. A partir de estudos de metanálise, longitudinais e cross-culturais, os autores apontam que os trabalhadores que apresentam desempenho superior são mais engajados no trabalho, exibem mais cidadania organizacional e menos comportamentos contraproducentes no trabalho (como o absenteísmo), em comparação aos menos engajados. Também há ampla evidência de que o engajamento está relacionado com atitudes positivas de comportamento organizacional e com o sucesso do negócio. Schaufel et al. (2009) demonstram que o aumento de demandas e diminuição de recursos prediz a exaustão no trabalho (Burnout) e se associa positivamente com a duração do absenteísmo por adoecimento. Já o aumento de recursos laborais (dentre estes, os recursos pessoais dos trabalhadores) prediz engajamento no trabalho e se associa negativamente com a frequência do absenteísmo por adoecimento. Não há dúvidas que o processo de pressão laboral desempenha um papel importante para as faltas no trabalho; este estudo destaca que o investimento em recursos laborais é fator protetivo ao absenteísmo por influenciar de modo positivo a motivação das pessoas na organização e realizar seu trabalho de modo prazeroso.

Na teoria JDR, há sempre um emparelhamento entre recursos (fatores motivacionais) e demandas (estressores) presentes na atividade laboral e que são associadas ao desempenho saudável mediado pelo engajamento. Nesse sentido, as intervenções positivas não devem subavaliar o papel de recursos e demandas laborais. Não adianta a organização preparar todo seu planejamento estratégico e se equipar de recursos, se estes últimos não se constituírem em fatores que irão impulsionar o 
comportamento de seus trabalhadores. O resultado de exigências elevadas sem recursos motivacionais é uma espiral descendente de motivação e de desempenho. Não serão campanhas motivacionais, com premiações pouco identificadas com as reais necessidades das pessoas, que resolverão o baixo engajamento ou a insatisfação.

O engajamento no trabalho é o funcionamento ótimo do trabalhador, ao passo que distress, síndrome de Burnout, monotonia, adição ao trabalho, dentre outros, são sintomas de acometimentos à sua saúde. Crawford, LePine e Rich (2010) demonstram que é possível diferenciar "demandas de obstáculos" ao desempenho advindos de conflitos interpessoais e de valores, ambiguidade de papéis ou insegurança laboral, das "demandas de desafio". Estas últimas estão diretamente ligadas à complexidade das tarefas, pressão por prazos e responsabilidades atribuídas ao trabalhador. São aspectos de contexto laboral (recursos e demandas) que devem ser analisados conjuntamente, a dinâmica entre eles é o aspecto-chave para compreensão do processo de engajamento no trabalho.

Nessa linha, Schaufeli et al. (2009) demonstram que o absenteísmo pode ser gerado por dois motivos principais. Primeiro, de forma voluntária, para se afastar de situações adversas no trabalho, tendo em vista que funcionários com menor satisfação e comprometimento organizacional faltam mais; visto na frequência das faltas. Ou, de modo involuntário, como uma reação ao estresse negativo (distress) causado por elevadas demandas que geram sobrecarga ou conflitos; que é identificado pela duração das faltas. O absenteísmo voluntário se associa ao processo motivacional, o que significa que tornar os recursos de trabalho suficientes tem o potencial de elevar a motivação dos trabalhadores e o engajamento no trabalho, reduzindo essas faltas. Já o absenteísmo involuntário se associa ao aumento das exigências que esgotam a energia dos trabalhadores, gerando afastamentos por saúde.

Tais achados permitem realizar diferentes predições sobre os tipos de absenteísmo pelo modelo JDR e sustentam a criação de estratégias positivas organizacionais, específicas para prevenção, promoção ou proteção à saúde dos trabalhadores. O central nessa análise é identificar a dinâmica entre recursos e demandas frente às motivações intrínsecas e bem-estar dos trabalhadores, processo que tem como consequente o desempenho destes na organização. $\mathrm{O}$ balanceamento entre essas variáveis deve buscar um funcionamento ótimo entre recursos e demandas, do ponto de vista das pessoas e da organização, concomitantemente.

Rhenen, Blonk, Schaufeli e Dijk (2007) diagnosticam que são necessários mais do que programas de prevenção de estresse organizacionais para diminuir substancialmente o absenteísmo. Embora tais ações organizacionais produzam intervenções cognitivas, físicas e de ensino de estratégias de coping aos trabalhadores com capacidade relativa de minorar o absenteísmo, seu efeito é transitório. Ações de prevenção não afetam variáveis fortemente associadas ao absenteísmo por adoecimento, por isso os autores destacam a importância do investimento nos recursos pessoais dos trabalhadores. Por outro lado, Consiglio, Borgogni, Alessandri e Schaufeli (2013) demonstram que a autoeficácia é mediadora entre recursos e demandas laborais na competência dos profissionais. Ao se aliar à esperança, ambas se apresentam como forças para obter metas específicas, que podem ser escolhidas ou rejeitadas pela avaliação que o indivíduo faz de si mesmo (autoeficácia) ou da expectativa de alcançá-las (esperança). Há outros recursos pessoais estudados nessa linha, tais como: autoestima, redesenho do trabalho, bem-estar, flow ou florescimento (Freitas \& Reis, 2019; Oliveira \& Ferreira, 2016).

Há que se observar, por fim, as bases teóricas dos pesquisadores, visto que há modelos diferentes para se interpretar o engajamento no trabalho (Schaufeli, 2018; Vazquez \& Schaufeli, 2019). A escala do engajamento no modelo JDR foi elaboradora por Schaufeli e Bakker (2004) e adaptada para o Brasil, por Vazquez et al. (2016) e Vazquez e Schaufeli (2019). No Brasil, há um conjunto de pesquisadores dedicados a estudar experiências subjetivas e potencialidades humanas, tendo adaptado escalas com qualidade psicométrica adequada (Hutz, 2014, 2016). De tudo que foi descrito, pode-se observar inúmeras evidências nos estudos em engajamento no trabalho com avanços em diferentes frentes. Certamente teremos desdobramentos e novas evidências empíricas no tema muito em breve.

\section{Os Comportamentos de Redesenho do Trabalho}

A perspectiva dos comportamentos de redesenho do trabalho (Petrou, Demerouti, Peeters, Schaufeli, \& Hetland, 2012; Tims \& Bakker, 2010; Wrzesniewski \& Dutton, 2001) dedica-se ao estudo das formas proativas pelas quais os empregados atuam em seu contexto laboral, de modo a identificar os problemas e resolvê-los (ainda que isso implique na alteração de rotinas), com o objetivo de tornar suas atividades mais ajustadas as suas preferências e necessidades (Tims, Bakker, \& Derks, 2013) e a organização mais adaptada a seu ambiente externo (Ghitulescu, 2013).

Tomando por base os resultados de pesquisas qualitativas realizadas com diferentes grupos ocupacionais, evidenciou-se que muitos dos trabalhadores participavam ativamente da reconfiguração de suas tarefas, sem receber, na maioria das vezes, suporte de seus superiores ou organizações, Wrzesniewski e Dutton (2001) introduziram a expressão "comportamentos de redesenho do trabalho (job crafting)" na literatura. Esses comportamentos consistem em iniciativas dos profissionais, sem a participação de seus superiores, com objetivo de promover mudanças físicas, cognitivas e relacionais nas tarefas e relações sociais mantidas no contexto do trabalho. Eles se referem, portanto, a alterações espontâneas e não 
supervisionadas que os indivíduos realizam no âmbito de suas próprias atividades laborais (Lyons, 2008).

As mudanças físicas dizem respeito a alterações no formato, no conteúdo e no escopo das tarefas assinaladas aos trabalhadores. Podem envolver, por exemplo, modificações em relação ao tempo dedicado a cada tarefa, à quantidade de tarefas e às rotinas. $\mathrm{O}$ trabalhador poderá envidar esforços para realizar suas tarefas habituais de novas formas, por sentir que seu trabalho está ficando monótono e repetitivo. As mudanças cognitivas associam-se às alterações no modo de perceber tarefas $\mathrm{e}$ relações de trabalho, como no caso de um vendedor de bilhetes de teatro, que passa a perceber sua tarefa como importante para a diversão do público. As mudanças relacionais são modificações na qualidade e na quantidade de interações que os trabalhadores mantêm com demais colegas de trabalho, quando pode oferecer ajuda a estes, como forma de interagir mais frequentemente.

Em síntese, o redesenho do trabalho consiste em comportamentos positivos e funcionais que objetivam a melhoria de tarefas e processos, muito embora não façam parte das descrições formais de cargos. A principal motivação subjacente é a de tornar o contexto do trabalho prescrito mais ajustado às preferências, competências e necessidades individuais, para ser mais significativo e capaz de contribuir para o desenvolvimento da identidade com o trabalho e com a carreira. Seu principal foco é o indivíduo, ainda que suas consequências possam, a depender do contexto, ser benéficas à organização. Nas situações em que as tarefas são pouco especificadas, podem ser benéficos para a organização, por estabelecer práticas de trabalho capazes de contribuir para desempenho mais eficiente (Ghitulescu, 2006; Wrzesniewski \& Dutton, 2001).

Nesse modelo, o comportamento de redesenho do trabalho surge em decorrência de três necessidades básicas: manter controle sobre o próprio ambiente de trabalho para evitar seus efeitos negativos, desenvolver autoestima positiva e manter interações com os demais colegas de trabalho. As consequências de tais comportamentos relacionam-se ao significado do trabalho e à obtenção de uma identidade laboral positiva, que contribuirão para a satisfação no trabalho e para o bem-estar físico e mental do trabalhador (Berg, Grant, \& Johnson, 2010).

O modelo preconiza, ainda, que as relações entre a motivação para a satisfação de necessidades básicas e comportamentos de redesenho do trabalho são moderadas por fatores situacionais e disposicionais. Fatores situacionais são as oportunidades para a emissão de comportamentos de redesenho, maiores quando há maior autonomia, menos interdependência entre tarefas e menos proximidade com a chefia imediata. Os fatores disposicionais dizem respeito à orientação do indivíduo acerca de seu trabalho e à sua orientação motivacional. A orientação para o trabalho vê a própria atividade laboral como um instrumento de recompensa financeira, a carreira como instrumento para o desenvolvimento pessoal ou a sociedade como fonte de prazer e de utilidade social (Wrzesniewski \& Dutton, 2001). Trabalhadores com orientação para a carreira poderão direcionar suas atividades de redesenho do trabalho para o aumento da interação com as pessoas com maior probabilidade de influenciarem sua ascensão profissional na organização. Já a orientação motivacional desdobra-se em intrínseca (desempenho orientado à satisfação de necessidades individuais) e extrínseca (desempenho orientado à satisfação de necessidades alheias ao indivíduo). Trabalhadores com motivações intrínsecas poderão se engajar mais frequentemente em comportamentos de redesenho do trabalho que lhes permitam expressar de forma plena suas competências.

Inspirados nessa linha, mas procurando não se restringir apenas à modificação de tarefas e relações, Tims et al. (Tims \& Bakker, 2010; Tims, Bakker, \& Derks, 2012) e Petrou et al. (2012) desenvolveram tipologias dos comportamentos de redesenho do trabalho no Modelo JDR (Taris \& Schaufeli, 2016), de modo a vincular tais comportamentos a um maior número de aspectos do contexto do trabalho e de ocupações. Tims e Bakker (2010) propõem que os comportamentos de redesenho do trabalho são mudanças proativas que os trabalhadores realizam para obter equilíbrio entre as demandas laborais, recursos de trabalho, suas habilidades e necessidades pessoais. Nesse sentido, procuram diminuir demandas e aumentar recursos do trabalho para galgarem melhor adaptação a ele (ajuste pessoa-trabalho) e maior motivação para alcançar suas metas de trabalho.

Para Tims et al. (2012), os comportamentos de redesenho do trabalho têm quatro dimensões: 1 . aumento dos recursos estruturais do trabalho, 2. aumento dos recursos sociais do trabalho, 3. aumento das demandas desafiadoras e 4. diminuição das demandas de obstáculo. Recursos estruturais são, para o incremento dos aspectos intrínsecos ao desenho do trabalho, como solicitações de mais autonomia, enquanto o aumento dos recursos sociais são os aspectos do contexto social em que o trabalho se desenvolve, como pedidos de mais feedback dos colegas e supervisores. Demandas desafiadoras são o esforço para maximizar as metas difíceis, como no caso de inserção em novos projetos, enquanto a diminuição das demandas de obstáculo são as tentativas de minimização de tarefas que impedem o alcance de metas. Tims et al. (2012) criaram a Escala de Comportamentos de Redesenho de Trabalho, adaptada para o Brasil por Chinelato, Ferreira e Valentini (2015).

Em perspectiva diferente, mas ainda referido no modelo JDR, Petrou et al. (2012) conceituam os comportamentos de redesenho do trabalho como ações voluntárias autoiniciadas, que objetivam a busca de recursos e de demandas desafiadoras, além da 
redução de demandas de obstáculo. Os autores congregam as categorias de aumento de recursos estruturais e de aumento de recursos sociais, da tipologia de Tims e Bakker (2010), em uma só, denominada de busca de recursos do trabalho. Nessa proposta, os comportamentos de redesenho do trabalho são ações diárias e não episódicas, que podem se manifestar em qualquer ambiente organizacional. Petrou et al. (2012) adaptaram a escala original de Tims et al. (2012) para uma escala de 13 itens e três fatores, com boas características psicométricas.

Comparando a perspectiva de Wrzesniewski e Dutton (2001) com as referenciadas ao modelo JDR (Petrou et al., 2012; Tims et al., 2012), Demerouti (2014) enfatiza que todas se focam em comportamentos para reconfigurar situações laborais, pelo enfrentamento dos problemas e busca de melhores soluções. Para a autora, as mudanças físicas (Wrzesniewski \& Dutton, 2001) guardam semelhança conceitual com as de demandas do trabalho (Tims et al., 2012; Petrou et al., 2012), enquanto as mudanças relacionais são conceitos similares às alterações nos recursos de trabalho. Contudo, as mudanças cognitivas (Wrzesniewski \& Dutton, 2001) não encontram correspondência em nenhuma categoria das duas tipologias no JDR, e são mais associadas a uma reconfiguração cognitiva de adaptação passiva ao contexto do trabalho do que a uma reação proativa (Bakker, Tims, \& Derks, 2012).

Investigações empíricas buscam elucidar antecedentes do redesenho do trabalho. Sobre os situacionais, há associações com: autonomia no desempenho da tarefa, complexidade das tarefas, pressão no trabalho, atividades desafiadoras, suporte da supervisão, maior posição hierárquica e mudanças organizacionais (Berg, Wrzesniewski, \& Dutton, 2010; Kira, Balkin, \& San, 2012; Leana, Appelbaum, \& Shevchuk, 2009; Lyons, 2008; Petrou et al., 2012). Já a interdependência das tarefas não tem se mostrado associada a tais comportamentos. Dos antecedentes individuais, verificou-se relação com: personalidade proativa, autoeficácia, temperamento de abordagem e foco regulatório de promoção ou orientado para autodesenvolvimento e desafios (Bakker et al., 2012; Bipp \& Demeroputi, 2014; Brenninkmeijer \& HekkertKoning, 2015; Tims, Bakker, \& Derks, 2014; Petrou \& Demerouti, 2015).

Cumpre ressaltar que o estudo dos comportamentos de redesenho do trabalho é bastante recente, tendo se acelerado somente nos últimos cinco anos. Questões sobre fatores contextuais e individuais que impactam as ações proativas e autoiniciadas estão sem respostas. Há carência de estudos sobre seus consequentes, bem como sobre moderadores e mediadores das relações, especialmente no contexto brasileiro. Pesquisas brasileiras sobre essa temática poderão servir para instrumentalizar os gestores das organizações brasileiras com ferramentas capazes de envolver seus colaboradores na criação de ambientes mais saudáveis e que atendam às suas necessidades e preferências. O que poderá se reverter em uma força de trabalho motivada e com melhores índices de desempenho individual e grupal.

\section{Considerações Finais}

O objetivo desse artigo teórico foi demonstrar a influência do movimento da Psicologia Positiva nas pesquisas organizacionais e do trabalho, por meio das evidências obtidas nas teorias de bem-estar, do engajamento no trabalho e dos comportamentos de redesenho. Nossa principal contribuição foi demonstrar o papel central desses construtos para o desenvolvimento científico com possíveis desdobramentos nas intervenções práticas producentes e saudáveis para o contexto organizacional e do trabalho.

Foi demonstrado, ao longo do texto, que as teorias que investigam o bem-estar no trabalho envolvem aspectos afetivos, cognitivos e psicológicos que atuam no ajustamento do indivíduo em diferentes domínios de suas vidas; sendo que em Psicologia Organizacional e do Trabalho estão associados ao desenvolvimento profissional, à qualidade de vida e à produtividade. Em conjunto, foram apresentados modelos teóricos e evidências que demonstram que o construto do engajamento no trabalho é um indicador de saúde do trabalhador e bem-estar laboral. E também se caracteriza como um fator-chave para o desempenho das pessoas nas organizações, por seu papel mediador na dinâmica do funcionamento ótimo dos recursos e demandas laborais. Finalmente, demonstrou-se que comportamentos de redesenho do trabalho são ações proativas individuais destinadas ao ajustamento do contexto do trabalho às preferências e necessidades do próprio trabalhador, o que lhe permite inovar, trazer para a organização novas rotinas práticas e desenvolver a criatividade no ambiente organizacional.

Nesse artigo foi defendido que o desenvolvimento da Psicologia Positiva baseada nas evidências de impacto do bem-estar, engajamento e redesenho no trabalho para o trabalhador e a própria organização pode influenciar os modelos de gestão e planejamento de intervenções organizacionais. As contribuições e avanços nesse campo do conhecimento permitirão o aprofundamento dos fenômenos que trazem à baila comportamentos dos trabalhadores que envolvem prazer, felicidade, inovação, proatividade e realização profissional. Estudos empíricos que testem as relações entre essas variáveis, em diferentes contextos, grupos ocupacionais e organizações de trabalho, são sugeridos. Também considera-se importante que sejam desenvolvidas pesquisas para testar o modelo teórico JDR em trabalhadores brasileiros. Assim, pelo olhar da Psicologia Positiva, somado ao conhecimento dos estudos tradicionais, se possibilitará a visão sistêmica sobre trabalho e as organizações com foco no bem-estar do trabalhador e no desempenho organizacional saudável. 


\section{Referências}

Bakker, A., \& Schaufeli, W. (2008). Positive organizational behavior: Engaged employees in flourishing organizations. Journal of Organizational Behavior, 29(2), 147-154. doi: 10.1002/job.515

Bakker, A. B., Tims, M., \& Derks, D. (2012). Proactive personality and job performance: The role of job crafting and work engagement. Human Relations, 65(10), 1359-1378. doi: 10.1177/0018726712453471

Berg, J. M., Wrzesniewski, A., \& Dutton, J. E. (2010). Perceiving and responding to challenges in job crafting at different ranks: When proactivity requires adaptivity. Journal of Organizational Behavior, 31(2-3), 158-186. doi: 10.1002/job.645

Berg, J. M., Grant, A. M., \& Johnson, V. (2010). When callings are calling: Crafting work and leisure in pursuit of unanswered occupational callings. Organizational Science, 21(5), 973-994. doi 10.1287/orsc.1090.0497

Bipp, T., \& Demerouti, E. (2014, October 8). Which employees craft their jobs and how? Basic dimensions of personality and employees' job crafting behaviour. Journal of Occupational and Organizational Psychology, 88(4), 631-655. doi: 10.1111/joop.12089

Brenninkmeijer, V., \& Hekkert-Koning, M. (2015). To craft or not to craft: The relationships between regulatory focus, job crafting and work outcomes. Career Development International, 20(2), 147-162. doi: 10.1108/CDI-12-2014-0162

Chinelato, R. S. C., Ferreira, M. C., \& Valentini, F. (2015). Evidence of validity of the Job Crafting Behaviors Scale. Paidéia, 25(62), 325-332. doi: 10.1590/1982-43272562201506

Consiglio, C, Borgogni, L, Alessandri, G., \& Schaufeli, W. B. (2013). Does self-efficacy matter for Burnout and sickness absenteeism? The mediating role of demands and resources at the individual and teams levels. Work \& Stress, 27(1), 22-42. doi: 10.1080/02678373.2013.769325.

Crawford, E, R., LePine, J., \& Rich, B. L. (2010). Linking job demands and resources to employee engagement and burnout: A theoretical extension and meta-analytic test. Journal of Applied Psychology, 95(5) 834-848. doi: 10.1037/a0019364

Dagenais-Demarais, V., Mendonça, H., Ferreira, M. C., \& Savoie, A. (2017). Pshychological well-being at work: Where are we and where do we go from here. Em Conceptualizing and assessing stress (pp. 65-84). Information Age Publishing (IAP).

Dagenais-Desmarais, V., \& Savoie, A. (2012). What is psychological well-being, really? A grassroots approach from organizational sciences. Journal of Happiness Studies, 13(4), 659-668. doi:10.1007/s10902-011-9285-3

Dalanhol, N. S., Machado, W. L., Pizarro, C. F., Hutz, C. S., \& Vazquez. A. C. (2017). Engajamento no trabalho, saúde mental e personalidade em oficiais de Justiça. PSICO-PUCRS, 48(2), 109-119. doi: 10.15448/1980-8623.2017.2.25885

Demerouti, E. (2014). Design your own job through job crafting. European Psychologist, 19, 237-247. doi: 10.1027/1016-9040/a000188

Diener, E., Oishi, S., \& Lucas, R. E. (2003). Personality, culture, and subjective well-being: Emotional and cognitive evaluations of life. Annual Review of Psychology, 54(1), 403-425. doi: 10.1146/annurev.psych.54.101601.145056

Efrom, C., \& Magnan, E. S. (2018). A saúde do trabalhador na perspectiva da Psicologia Positiva Organizacional e do Trabalho. Em Vazquez, A. C. S. \& Hutz, C. S. (Eds.). Aplicações da psicologia positiva. Trabalho e organizações. (pp. 63-82) São Paulo: Hogrefe.

Efrom, C., Vazquez, A., \& Hutz, C. (2019). Avaliação de fatores psicossociais no trabalho. Em C. Hutz, D. Bandeira, C. Trentini \& A. C. S. Vazquez (Eds). Avaliação Psicológica no Contexto Organizacional e do Trabalho (pp. 19-37). Porto Alegre: Artmed.

Fredrickson, B. L., \& Losada, M. F. (2005). Positive affect and the complex dynamics of human flourishing. The American psychologist, 60(7), 678-686. doi: 10.1037/0003-066X.60.7.678

Freitas, C. P. P., \& Reis, M. (2019). Recursos pessoais no trabalho: Definição, impacto e estratégicas para avalia-los. Em C. Hutz, D. Bandeira, C. Trentini \& A. C. S. Vazquez (orgs). Avaliação Psicológica no Contexto Organizacional e do Trabalho (pp.54-67). Porto Alegre: Artmed.

Ghitulescu, B. E. (2006). Shaping tasks and relationships at work: Examining the antecedents and consequences of employee job crafting. University of Pittsburgh.

Ghitulescu, B. E. (2013). Making change happen: The impact of work context on adaptive and proactive behavior. Journal of Applied Behavioral Science, 49(2), 206-245. doi: 10.1177/0021886312469254

Hutz, C. (2014). Avaliação em Psicologia Positiva. Porto Alegre: Artmed.

Hutz, C. (2016). Avaliação em Psicologia Positiva. Métodos e técnicas. São Paulo: Hoegrefe.

Hutz, C. S., Zanon, C., \& Brum Neto, H. (2013). Adverse working conditions and mental illness in poultry slaughterhouses in southern Brazil. Psicologia: Reflexão e Crítica, 26(2), 296-304. doi: 10.1590/S0102-79722013000200009

Kashdan, T. B., Biswas-Diener, R., \& King, A. (2008). Reconsidering happiness: the costs of distinguishing between hedonics and Eudaimonia. The Journal of Positive Psychology: Dedicated to Furthering Research and Promoting Good Practice, 3(4), 219-233. doi: $10.1080 / 17439760802303044$

Kira, M., Balkin, D. B., \& San, E. (2012). Authentic work and organizational change: Longitudinal evidence from a merger. Journal of Change Management, 12(1), 31-51. doi: 10.1080/14697017.2011.652374

Koukoulaki, T. (2014). The impact of lean production on musculoskeletal and psychosocial risks: An examination of sociotechnical trends over 20 years. Applied Ergonomics, 45(2), 198-212. doi: 10.1016/j.apergo.2013.07.018

Leana, C., Appelbaum, E., \& Shevchuk, I. (2009). Work process and quality of care in early childhood education: The role of job crafting. Academy of Management Journal, 52(6), 1169-1192. doi: 10.5465/amj.2009.47084651

Li, Z., Yin, X., Jiang, S., Wang, M., \& Cai, T. (2014). Psychological mechanism of subjective well-being: A stable trait or situational variability. Social Indicators Research, 118(2), 523-534. doi: 10.1007/s11205-013-0449-x

Lyons, P. (2008). The crafting of jobs and individual differences. Journal of Business Psychology, 23(1-2), 25-36. doi: 10.1007/s10869-008-9080-2

Magnavita, N. (2012). Validation of the Italian version of the HSE Indicator Tool. Occupational Medicine, 62(4), 288-94. doi: 10.1093/occmed/ kqs025

Mazloumi, A., Rostamabadi A., Nasl Saraji G., \& Rahimi Foroushani, A. (2012). Work ability index (WAI) and its association with psychosocial factors in one of the petrochemical industries in Iran. Journal of Occupational Health, 54(2), 112-118. doi: 10.1539/joh.110150-OA

Mendonça, H., Ferreira, M. C., Caetano, A., \& Torres, C. V. (2014). Cultura organizacional, coping e bem-estar subjetivo: Um estudo com professores de universidades brasileiras. Revista Psicologia: Organizações e Trabalho, 14(2), 230-244.

Mendonça, H., \& Ferreira, M. C. (2018). Florescimento no trabalho (Flourishing): Novo conceito, velho fenômeno. Em A. C. S. Vazquez \& C. S. Hutz, (Eds.). Aplicações da Psicologia Positiva. Organizações e Trabalho (pp. 187-200). São Paulo: Hogrefe. 
Mendonça, H., \& Silva, A. J. (2019). Avaliação do bem-estar no trabalho. Em C. Hutz, D. Bandeira, C. Trentini \& A. C. S. Vazquez (Eds.). Avaliação Psicológica no Contexto Organizacional e do Trabalho (pp. 68-85). Porto Alegre: Artmed.

Nery, V. F., Neiva, E. R., \& Mendonça, H. (2016). The Changing Context and the organizational Justice Impact on the Employee WellBeing. Paidéia, 26(65), 317-324. doi:10.1590/1982-43272665201609

Oliveira, D. F., \& Ferreira, M. C. (2016). O impacto das percepções de justiça organizacional e da resiliência sobre o engajamento no trabalho. Estudos de Psicologia, 33(4), 747-755. doi: 10.1590/1982-02752016000400017

Petrou, P., \& Demerouti, E. (2015). Trait-level and week-level regulatory focus as a motivation to craft a job. Career Development International, 20(2), 102-118. doi: 10.1108/CDI-09-2014-0124

Petrou, P., Demerouti, E., Peeters, M. C. W., Schaufeli, W. B., \& Hetland, J. (2012). Crafting a job on a daily basis: Contextual antecedents and the effect of work engagement. Journal of Organizational Behavior, 33(8), 1120-1141. doi: 10.1002/job.1783

Reijseger, G., Schaufeli, W. B., Peeters, M. C. W., \& Taris, T. W. (2012). Ready, set, go! A model of the relation between work engagement and performance. Em S. P. Gonçalves \& J.G. Neves (Eds.). Occupational health psychology: From burnout to well-being (pp. 289-306). Scientific \& academic publishing: USA.

Rhenen, W., Blonk, R. W. B, Schaufeli, W. B., \& Dijk, F. J. H. (2007). Can sickness absence be reduced by stress reduction programs: on the effectiveness of two approaches. International Archives of Occupational Environmental Health, 80(6), 505-515. doi: 10.1007/s00420-0060157-9

Ryff, C. (1989). Happiness is everything, or is it? Explorations on the meaning of psychological well-being. Journal of Personality and Social Psychology, 57(6), 1069-1081.

Salanova, M., del Libano, M., Llorens, S., \& Schaufeli, W. B. (2014). Engaged, workaholic, burned-out or just 9-to-5? Toward a typology of employee well-being. Stress \& Health, 30(1), 71-81. doi: 10.1002/smi.2499

Schaufeli, W. B. (2018). O que é engajamento? Em Claudio S. Hutz \& Ana C. S. Vazquez (Eds.), Aplicações da Psicologia Positiva. Organização e Trabalho (pp. 33-62). São Paulo: Hoegrefe.

Schaufeli, W. B., \& Bakker, A.B. (2004). Job demands, job resources, and their relationship with burnout and engagement: A multi-sample study. Journal of Organizational Behavior, 25(3), 293-315. doi: 10.1002/job.248

Schaufeli, W. B., Bakker, A. B., \& Van Rhenen, W. (2009). How changes in job demands and resources predict burnout, work engagement, and sickness absenteeism. Journal of Organizational Behavior, 30(7), 893-917. doi: 10.1002/job.595

Simmons, B L. (2014). Organizational characteristics of happy organizations. Em Peter Y. Chen \& Cary L. Cooper. Wellbeing: A complete reference guide, Work and wellbeing (pp. 139-156). UK: Wiley Brackwell.

Taris, T. W., \& Schaufeli, W. B. (2016). The Job Demands-Resources model. Em S. Clarke, T. M. Probst, F. Guldenmund \& J. Passmore (Eds.). The Wiley Blackwell handbook of the psychology of occupational safety and workplace health (pp. 157-180). Chichester: John Wiley.

Timms, C., \& Brough, P. (2013). 'I like being a teacher'. Career satisfaction, the work environment and work engagement. Journal of Educational Administration, 51(6), 768-789. doi: 10.1108/JEA-06-2012-0072

Tims, M., \& Bakker, A. B. (2010). Job crafting: Towards a new model of individual job redesign. South African Journal of Industrial Psychology, 36(2), 1-9.

Tims, M., Bakker, A. B., \& Derks, D. (2012). Development and validation of the job crafting scale. Journal of Vocational Behavior, 80(1), 173186. doi: 10.1016/j.jvb.2011.05.009

Tims, M., Bakker, A. B., \& Derks, D. (2014). Daily job crafting and the self-efficacy - performance relationship. Journal of Managerial Psychology, 29(5), 490-507. doi: 10.1108/JMP-05-2012-0148

Tims, M., Bakker, A. B., \& Derks, D. (2013). The impact of job crafting on job demands, job resources, and well-being. Journal of Occupational Health Psychology, 18(2), 230-240.

Vazquez, A. (2018). A psicologia positiva organizacional e do trabalho (PPOT): Fundamentos e aplicações. Em A. C. S. Vazquez \& C. S. Hutz. (Eds.). Aplicações da Psicologia Positiva. Trabalho e Organizações (pp. 5-32). São Paulo: Hogrefe.

Vazquez, A., Pianezolla, M., \& Hutz, C. (2018). Assessment of psychosocial factors at work: A systematic review. Estudos de Psicologia, 35(1), 5-13. doi: 10.1590/1982-02752018000100002

Vazquez, A., \& Schaufeli W. (2019). Contribuições da Psicologia Positiva para a área organizacional e do Trabalho. Em C. T. Reppold, \& L. S. Almeida. (Eds.). Psicologia Positiva: Educação, saúde e trabalho (pp. 105-134). Porto: CERPSI.

Violanti, J., Mnatsakanova, A., Andrew, M. E., Allison, P., Gu, J. K., \& Fekedulegn, D. (2018). Effort-Reward Imbalance and Overcommitment at Work: Associations with Police Burnout. Police Quaterly, 21(4), 440-460. doi: 10.1177/1098611118774764

Weiss, H., \& Cropanzano, R. (1996). Affective events theory: A theoretical discussion. Research in Organizational Behavior, 18(1), 1-74.

Wrzesniewski, A., \& Dutton, J. D. (2001). Crafting a Job: Revisioning employees as active crafters of their work. The Academy of Management Review, 26(2), 179-201. doi: 10.5465/amr.2001.4378011

recebido em agosto de 2019 aprovado em outubro de 2019

\section{Sobre as autoras}

Ana Cláudia Souza Vazquez é psicológica e professora na Universidade Federal de Ciências da Saúde de Porto Alegre (UFCSPA), mestre em Saúde Coletiva pela UERJ e doutora em Administração pela UFRGS, com MBA na área de gestão de pessoas pela USP. Atualmente é Pró-Reitora de Gestão com pessoas na UFCSPA e presidente da Associação Brasileira de Psicologia Positiva.

Helenides Mendonça é psicóloga da Pontifícia Universidade Católica de Goiás (PUC-GO), mestre em Filosofia pela Política pela Universidade Federal de Goiás e doutora em Psicologia pela Universidade de Brasília, com pós-doutorado no Instituto Universitário de Lisboa - ISCTE/IUL. Atualmente é vice-presidente da Associação Brasileira de Psicologia Positiva.

Maria Cristina Ferreira é psicóloga pela Universidade do Estado do Rio de Janeiro (UERJ), mestre e doutora em Psicologia pela Fundação Getúlio Vargas - RJ, na área de Psicologia Cognitiva, com estágio pós-doutoral realizado na Victoria University at Wellington. Atualmente é coordenadora do Programa de Pós-graduação em Psicologia da UNIVERSO-RJ. 